\title{
Expresión Inmunohistoquímica de Homólogo de Fosfatasa y Tensina en Glioblastoma Multiforme y su Relación con Sobrevida
}

\author{
Immunohistochemical Expression of Phosphatase and Tensin Homologue \\ in Glioblastoma Multiforme and its Relationship to Survival
}

Esteban F. Espinoza-García ${ }^{\text {; }}$ Andrés Horlacher-Kunstmann² \& Francisco M. González-Vicuña²

\begin{abstract}
ESPINOZA-GARCÍA, E. F.; HORLACHER-KUNSTMANN, A. \& GONZÁLEZ-VICUÑA, F. M. Expresión inmunohistoquímica de homólogo de fosfatasa y tensina en glioblastoma multiforme y su relación con sobrevida. Int. J. Morphol., 37(3):800-804, 2019.

RESUMEN: El glioblastoma multiforme es el subtipo de gliomas más frecuente en adultos, con una pobre sobrevida promedio posterior al diagnóstico incluso si se aplica el tratamiento óptimo. Se ha estudiado marcadores tumorales de buen pronóstico, siendo controversial la expresión del homólogo de fosfatasa y tensina. Se estudió muestras parafinadas obtenidas de pacientes con glioblastoma multiforme en el Hospital Carlos Van Buren de Valparaíso, Chile, entre 2010 y 2014. Se realizó análisis inmunohistoquímico para expresión de homólogo de fosfatasa y tensina, estudiándose la intensidad y el patrón de expresión en astrocitos y células epiteliales, además de revisión de datos clínicos. Análisis estadístico utilizando SPSS v20. Se estudió la expresión de PTEN en 21 pacientes. Un 52,4 $\%$ presentó una baja expresión en núcleos de astrocitos, con un promedio de sobrevida de 14,2 meses comparado con 10,2 meses del grupo con alta expresión $(\mathrm{p}=0,33)$. Se encontró una intensa expresión endotelial en tejido tumoral, comparado con tejido cerebral sin tumor. Se encontró una relación entre la expresión nuclear en astrocitos con diferencias en el tiempo de sobrevida, aunque no estadísticamente significativa, requiriéndose nuevos estudios para corroborarlo. La intensa expresión endotelial observada en tejido tumoral debe ser analizada de forma dirigida.
\end{abstract}

PALABRAS CLAVE: Glioblastoma; Glioma; Inmunohistoquímica; Neoplasias Cerebrales; Sobrevida.

\section{INTRODUCCIÓN}

El glioblastoma multiforme es el subtipo más común y más letal de gliomas, cuya incidencia se describe entre 0,6 a 3,7 por 100.000 personas, siendo el tercer tipo tumoral más frecuentemente reportado, luego de los meningiomas y los tumores pituitarios (Gousias et al., 2009; Lee et al., 2010; Dobes et al., 2011; Gigineishvili et al., 2013; Ostrom et al., 2013), considerado uno de los tumores más letales en humanos (Dunn et al., 2012). Representa hasta el $50 \%$ de todos los gliomas cerebrales primarios, y su pronóstico continúa resultando sombrío (Ohgaki et al., 2004; Stupp et al., 2005). Se describe un porcentaje de sobrevida de $0,05 \%$ a $4,7 \%$ a 5 años posterior al diagnóstico (Ostrom et al., 2014) y un tiempo de sobrevida promedio de 12 a 15 meses con el tratamiento óptimo descrito, el que incluye cirugía resectiva, quimioterapia con temozolomida y radioterapia (Stupp et al.). Sin tratamiento, la mayoría de estos pacientes fallece en un período de 3 meses (Hwang et al., 2014). Esta enfermedad afecta comúnmente a la población adulta, siendo el rango de edad más frecuentemente afectado entre 45 a 70 años, con ligera predominancia masculina (Ohgaki \& Kleihues, 2005). Este tipo tumoral se puede desarrollar a partir de un glioma de menor grado, como un glioma de bajo grado (grado II de la OMS) o de un astrocitoma anaplásico (grado III de la OMS), sin embargo, la mayoría se desarrolla de novo sin evidencia de una lesión preexistente (Louis et al., 2007).

El homólogo de fosfatasa y tensina (PTEN) es un supresor tumoral que actúa sobre el fosfatidilinositol $(3,4,5)$-trifosfato, PtdIns $(3,4,5) \mathrm{P} 3$, producto mayor de la PI 3-kinasa, que provoca un aumento en la actividad apoptótica (Yamada \& Araki, 2001). La significancia como valor pronóstico del PTEN en glioblastoma es todavía controversial. A pesar de que múltiples estudios sugieren que no existe relación entre mutaciones del PTEN y la sobrevida o quimiosensibilidad, otros estudios han rela-

\footnotetext{
${ }^{1}$ Unidad de Neurocirugía. Hospital San Camilo, San Felipe, Chile.

2 Servicio de Neurocirugía. Hospital Carlos Van Buren, Valparaíso, Chile.

Este trabajo se financió con aportes entregados por Laboratorio Saval, adjudicados mediante concurso "Beca de Investigación Saval", no teniendo la corporación injerencia ni en la idea del proyecto, ni en la creación del protocolo de estudio ni en el análisis de los resultados.
} 
ESPINOZA-GARCÍA, E. F.; HORLACHER-KUNSTMANN, A. \& GONZÁLEZ-VICUÑA, F. M. Expresión inmunohistoquímica de homólogo de fosfatasa y tensina en glioblastoma multiforme y su relación con sobrevida. Int. J. Morphol., 37(3):800-804, 2019.

cionado la pérdida de función del PTEN con un comportamiento más agresivo (Xu et al., 2014). En un estudio, se estudió la expresión del PTEN en 25 muestras de gliomas, evidenciándose relación entre la expresión, el grado tumoral y el tiempo de sobrevida (Ermoian et al., 2002). En otro estudio, utilizando 129 muestras de pacientes operados por glioblastoma multiforme, se estudió la expresión de PTEN y su relación con sobrevida, encontrándose diferencias estadísticamente significativas a favor de los pacientes sin pérdida de PTEN, con un período de sobrevida de 166 días vs 437 días (Bäcklund et al., 2003). En contraste, se revisó en otro estudio un total de 586 casos de GBM, encontrándose una relación entre la sobreexpresión del PTEN y una menor sobrevida ( $\mathrm{Xu}$ et al.).

Algunos estudios no han encontrado relación entre la expresión del PTEN y el pronóstico. En un estudio publicado por Kraus et al. (2000), no se encontró relación entre la sobrevida y varios factores biomoleculares, entre ellos la expresión del PTEN. Por otra parte, (Yang et al., 2008) trataron de encontrar una respuesta favorable del RAD001, un inhibidor de mTOR, en base a que la vía PI3K/Akt/mTOR se encontraría activada con la pérdida del PTEN, no pudiéndose predecir la respuesta a este fármaco según el perfil de PTEN. Carico et al. (2012) tampoco pudieron encontrar una relación entre la expresión del PTEN y la sobrevida en GBM diagnosticados de novo, estudiándose 188 pacientes con este propósito.

A partir de lo anterior, se diseñó un estudio cuyo objetivo principal fue describir y comparar la sobrevida global de los pacientes según los resultados obtenidos con la técnica inmunohistoquímica de expresión de homólogo de fosfatasa y tensina de la cohorte analizada.

\section{MATERIAL Y MÉTODO}

Se realizó un estudio analítico de cohorte retrospectivo, estudiándose las muestras parafinadas de pacientes operados con diagnóstico de Glioblastoma Multiforme OMS IV en el Hospital Carlos Van Buren, Valparaíso, Chile, entre los años 2010 y 2014, con edad entre 18 y 70 años, esto último para que los resultados fueran comparables con resultados internacionales. Se descartaron pacientes con índice de ECOG postoperatorio superior a 2. Para esto, se utilizó los registros de Anatomía Patológica para identificar las muestras de pacientes con diagnóstico de glioblastoma multiforme OMS IV y realizar el estudio de sobrevida posterior. Las muestras de tejido tumoral fueron extraídas del Servicio de Anatomía Patológica para su posterior estudio biomolecular.
El estudio mediante inmunohistoquímica se realizó en la Unidad de Anatomía Patológica del Hospital Carlos Van Buren, Valparaíso, Chile. Se realizó secciones de tejido de $4 \mathrm{~mm}$, las cuales fueron montadas en portaobjeto cargado positivamente (Superfrost Plus, Thermo Scientific). Se utilizó el kit EnVision FLEX, Mouse, High pH (DAKO), según instrucciones de fabricante, utilizando anticuerpo antiPTEN (Abcam Inc., Cod. AB170941). Para control de tinción, se realizó el mismo proceso en tejido cerebral de autopsia, disponible en banco de tejidos del Hospital Carlos Van Buren. El análisis de tinción fue realizado por dos observadores independientes, anatomopatólogos. Se consignó cualitativamente la expresión celular en 4 grados ( 0 : ausente, I: leve, II: moderada, III: intensa) y se consignó además el porcentaje de células con expresión de PTEN. Lo anterior se realizó para las células endoteliales y los astrocitos, diferenciándose además el grado de expresión en núcleo y en el citoplasma de estas células. Para clasificar en alta o baja expresión, se realizó una escala de puntuación semicuantitativa según grado de expresión y según porcentaje de células con expresión de PTEN. De acuerdo con el grado de expresión, se otorgó un puntaje desde 0 a 3 puntos si presentaba ausencia de expresión, expresión leve, moderada o intensa respectivamente, según la evaluación por dos anatomopatólogos independientes. Según el porcentaje de células con expresión de PTEN, se otorgó desde 1 a 4 puntos si este porcentaje de expresión se encontraba entre 0-25 $\%, 26-50 \%, 51-75 \%$ y sobre $75 \%$ respectivamente. Según la sumatoria de estos dos factores, se clasificó como alta expresión un puntaje mayor o igual a 4 puntos, y como baja expresión un puntaje de 3 o menor. Se realizó el estudio de registros médicos de los pacientes para consignar tiempo de sobrevida y características clínicas. Ya que la decisión terapéutica fue determinada por el médico tratante, se consignó si ésta correspondió a biopsia, resección parcial o resección completa, corroborada mediante imagenología postoperatoria. Se analizó la sobrevida global en meses y se determinó el porcentaje de sobrevida al año y a los dos años, estratificándose de acuerdo a expresión baja o alta de PTEN, además del patrón inmunohistoquímico de expresión. En la construcción de la base de datos, se asignó un número de acuerdo al orden de la biopsia procesada por año, evitando incuir datos que pudieran identificar al paciente, manteniéndose su anonimato. Se realizó una base de datos utilizando Microsoft巴 Excel. La sobrevida global se midió a partir de la cirugía hasta el fallecimiento o último día de seguimiento, estudiándose con curvas de Kaplan-Meier y evaluando las diferencias entre ellas con test de log-rank, con intervalo de confianza de $95 \%$. Para el análisis multivariado se utilizó riesgo proporcional de Cox, evaluando el valor pronóstico de la expresión de PTEN. Se consideró valores significativos con p menores a 0,05 . Todos los cálculos fueron realizados utilizando el software IBM SPSS Statistics versión 
ESPINOZA-GARCÍA, E. F.; HORLACHER-KUNSTMANN, A. \& GONZÁLEZ-VICUÑA, F. M. Expresión inmunohistoquímica de homólogo de fosfatasa y tensina en glioblastoma multiforme y su relación con sobrevida. Int. J. Morphol., 37(3):800-804, 2019.

20. Se solicitó consentimiento informado a los pacientes vivos (o familiares) de los pacientes ingresados en este estudio. Este estudio fue evaluado por el Comité de Bioética de la Facultad de Medicina de la Universidad de Valparaíso, Chile (Acta de Evaluación Bioética No. 43/2015, Adenda No. 1/2016), y por el Comité Ético-Científico del Servicio de Salud Valparaíso-San Antonio, Chile (Acta de Aprobación $\mathrm{N}^{\mathrm{0}} 73$ ), siendo aprobado el protocolo de estudio por ambos comités. Este estudio se realizó con aportes otorgados por Laboratorio Saval, adjudicados mediante concurso "Beca de Investigación Saval", no teniendo injerencia la corporación ni en la idea del proyecto, ni en la creación del protocolo de estudio ni en el análisis de los resultados.

\section{RESULTADOS}

Relación entre el tiempo de sobrevida y el tiempo de sobrevida libre de progresión con respecto a la expresión del PTEN en tejido tumoral.

Se realizó una diferenciación entre la expresión endotelial y la expresión en astrocitos de PTEN, estudiándose el grado de expresión citoplasmática y nuclear en ambos grupos celulares (Fig. 1). Un hallazgo notorio fue que, con respecto a la expresión en células endoteliales, fue intensa en núcleo y en citoplasma sin existir diferencias entre las muestras tumorales, en comparación con la expresión de PTEN en célula endotelial de cerebro sin tumor que fue nula.

En la evaluación de la expresión de PTEN en astrocitos, se encontró que en el citoplasma no hubo gran- des variaciones entre las muestras tumorales, siendo esta expresión leve en tejido cerebral sin tumor. Donde se encontró diferencias fue en la expresión nuclear de PTEN, donde se evidenciaron 11 muestras con baja expresión de PTEN $(52,4 \%)$ y 10 con alta expresión (47,6 \%), siendo el promedio de sobrevida de 14,2 meses y de 10,2 meses respectivamente, sin diferencias estadísticamente significativas con el test de log-rank ( $\mathrm{p}=0,33$ ) (Fig. 2). El promedio de sobrevida libre de progresión en las muestras con baja expresión de PTEN fue de 11,4 meses, y en las muestras con alta expresión fue de 5,2 meses $(\mathrm{p}=0,23)$.

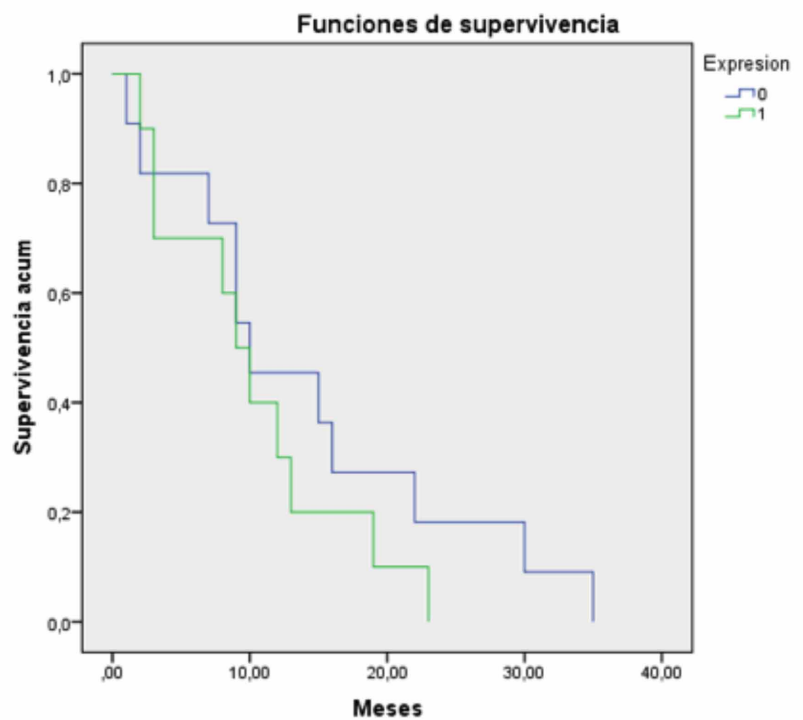

Fig. 2. Curva de Kaplan-Meier de sobrevida en relación a grado de expresión nuclear astrocitaria de PTEN (0: baja expresión; 1: alta expresión).
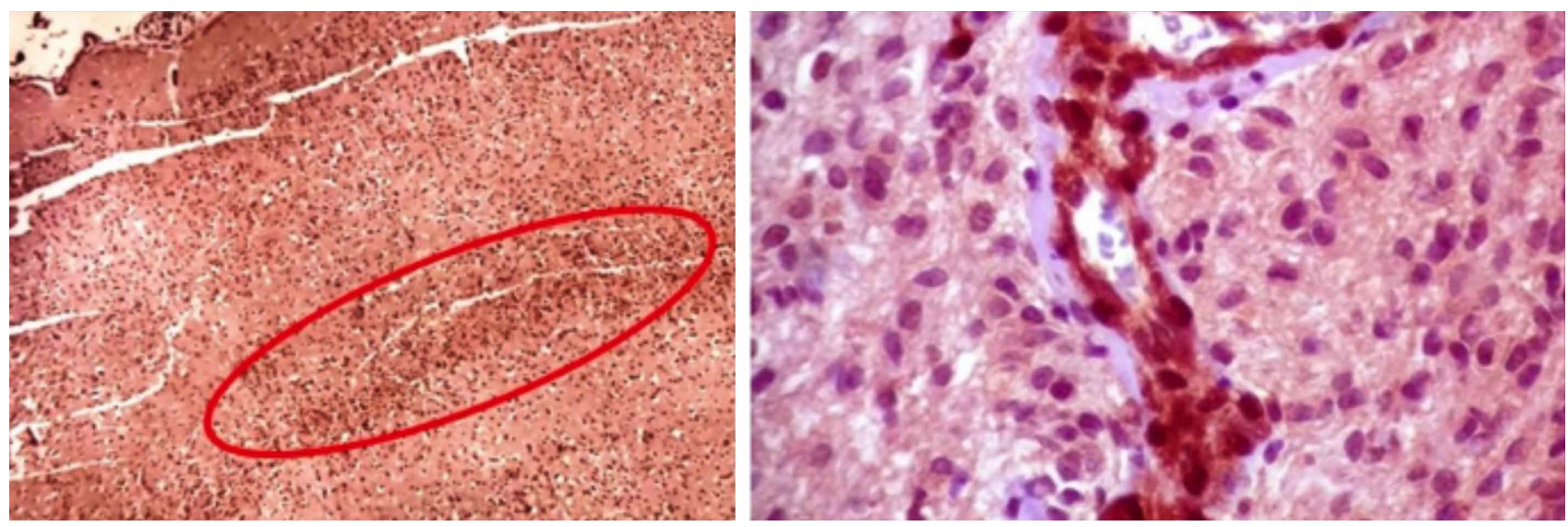

Fig. 1. A la izquierda se observa tinción en cerebro de autopsia, evidenciando expresión de PTEN a nivel nuclear de astrocitos en capa VI, sin tinción en resto de las capas de la sustancia gris, con expresión ausente del endotelio. A la derecha se observa la tinción en cerebro con diagnóstico de glioblastoma multiforme, con endotelio fuertemente positivo a nivel nuclear y citoplasmático y expresión moderada en citoplasma de astrocitos, patrón repetitivo en las muestras estudiadas en el presente trabajo. 


\section{DISCUSIÓN}

En Chile, no existen estudios nacionales que evalúen la relación entre la expresión de PTEN en glioblastoma y el tiempo de sobrevida, aunque fue evaluada la expresión en cáncer ovárico asociado a endometriosis por Castiblanco et al. (2006). En un estudio realizado en India por Srividya et al. (2011), se reportó que la deleción homocigótica 10q23/ PTEN se asociaba a peor pronóstico, con una media de sobrevida de 14 meses para el subgrupo con deleción contra 24 meses del grupo sin deleción, diferencia estadísticamente significativa. En otro estudio realizado por Smith et al. (2001) en población estadounidense, la alteración del PTEN no se relacionó estadísticamente con una diferencia en la sobrevida en pacientes con glioblastoma multiforme (11.7 meses con PTEN alterado y 11.2 en aquellos sin alteración, $\mathrm{p}=0.74$ ), aunque sí en pacientes con astrocitoma anaplásico. En el trabajo de Umesh et al. (2009), se encontró que la pérdida de expresión de PTEN pesquisada mediante inmunohistoquímica se correlacionaba con un menor tiempo de sobrevida en comparación con los pacientes con ausencia de esta alteración (12 meses vs 17 meses), aunque sin significancia estadística $(p=0,42)$. En nuestro trabajo, no encontramos diferencias estadísticamente significativas en cuanto a sobrevida o a sobrevida libre de progresión, e incluso, estas diferencias se encuentran a favor de que la disminución de la expresión pudiera ser en nuestra población un factor que aumente la sobrevida, lo que es contrario a lo que esperábamos encontrar. Para poder evaluar esto, se requieren mayores estudios, idealmente utilizando técnicas más avanzadas, como hibridación fluorescente in vitro, para poder determinar el rol real de este factor en la sobrevida. Un hallazgo interesante fue el encontrar patrones de expresión inmunohistoquímicos distintos en tejido tumoral y tejido no tumoral de la misma muestra, abriéndonos una puerta de investigación para evaluar la implicancia de este factor y de la vía PI3K/Akt/mTOR en la progresión de la enfermedad.

\section{AGRADECIMIENTOS}

Agradecemos la colaboración de los anatomopatólogos del Hospital Carlos Van Buren de Valparaíso, Dr. Carlo Lozano y Dr. Humberto Vallejos.

ESPINOZA-GARCÍA, E. F.; HORLACHER-KUNSTMANN, A. \& GONZÁLEZ-VICUÑA, F. M. Immunohistochemical expression of phosphatase and tensin homologue in glioblastoma multiforme and its relationship to survival. Int. J. Morphol., 37(3):800-804, 2019.
SUMMARY: Glioblastoma multiforme is the most frequent glioma subtype in adults, with poor survival rate after diagnosis even applying the optimal treatment. Tumoural markers have been studied looking for good prognosis, being the phosphatase and tensin homologue controversial. Paraffined samples were used from Carlos Van Buren Hospital in Valparaíso, Chile, between 2010 and 2014. An immunohistochemical analysis was performed looking for phosphatase and tensing homologue expression, studying the intensity and expression pattern in astrocytes and epithelial cells, in addition to clinical data. Statistical analysis was performed using SPSS v20. It was studied the phosphatase and tensin homologue expression in 21 patients. In the study, $52,4 \%$ presented low expression in astrocytic glial cell nuclei, with a survival mean of 14.2 months in comparison to 10.2 months in the high expression group $(\mathrm{p}=0.33)$. A very intense endothelial expression was found in tumoural tissue, in comparison to the tissue without tumor. A relation between nuclear expression in astrocytes and survival rate was found, although no statistically significant. The intense endothelial expression seen in tumoural tissue must be studied directly.

KEY WORDS: Brain neoplasms; Glioblastoma; Glioma; Immunohistochemistry; Survival.

\section{REFERENCIAS BIBLIOGRÁFICAS}

Bäcklund, L. M.; Nilsson, B. R.; Goike, H. M.; Schmidt, E. E.; Liu, L.; Ichimura, K. \& Collins, V. P. Short postoperative survival for glioblastoma patients with a dysfunctional Rb1 pathway in combination with no wild-type PTEN. Clin. Cancer Res., 9(11):4151-8, 2003.

Carico, C.; Nuño, M.; Mukherjee, D.; Elramsisy, A.; Dantis, J.; Hu, J.; Rudnick, J.; Yu, J. S.; Black, K. L.; Bannykh, S. I.; et al. Loss of PTEN is not associated with poor survival in newly diagnosed glioblastoma patients of the temozolomide era. PLoS One, 7(3):e33684, 2012.

Castiblanco, G. A.; Pires, N. Y.; Wistuba, O. I.; Riquelme, S. E.; Andrade, M. L. \& Corvalán, R. A. Rol patogénico del gen supresor de tumores PTEN en cáncer ovárico asociado a endometriosis. Rev. Med. Chile, 134(3):271-8, 2006.

Dobes, M.; Khurana, V. G.; Shadbolt, B.; Jain, S.; Smith, S. F.; Smee, R.; Dexter, M. \& Cook R. Increasing incidence of glioblastoma multiforme and meningioma, and decreasing incidence of Schwannoma (20002008): Findings of a multicenter Australian study. Surg. Neurol. Int., $2: 176,2011$.

Dunn, G. P.; Rinne, M. L.; Wykosky, J.; Genovese, G.; Quayle, S. N.; Dunn, I. F.; Agarwalla, P. K.; Chheda, M. G.; Campos, B.; Wang, A.; et al. Emerging insights into the molecular and cellular basis of glioblastoma. Genes Dev., 26(8):756-84, 2012.

Ermoian, R. P.; Furniss, C. S.; Lamborn, K. R.; Basila, D.; Berger, M. S.; Gottschalk, A. R.; Nicholas, M. K.; Stokoe, D. \& Haas-Kogan, D. A. Dysregulation of PTEN and protein kinase $\mathrm{B}$ is associated with glioma histology and patient survival. Clin. Cancer Res., 8(5):1100-6, 2002.

Gigineishvili, D.; Shengelia, N.; Shalashvili, G.; Rohrmann, S.; Tsiskaridze, A. \& Shakarishvili, R. Primary brain tumour epidemiology in Georgia: first-year results of a population-based study. J. Neurooncol., 112(2):241-6, 2013.

Gousias, K.; Markou, M.; Voulgaris, S.; Goussia, A.; Voulgari, P.; Bai, M.; Polyzoidis, K.; Kyritsis, A. \& Alamanos, Y. Descriptive epidemiology of cerebral gliomas in northwest Greece and study of potential predisposing factors, 2005-2007. Neuroepidemiology, 33(2):89-95, 2009. 
Hwang, B.; Chaichana, K. \& Quiñones-Hinojosa, A. The Role of Surgery in the Management of High-Grade Gliomas (Newly Diagnosed, Recurrent, and Multifocal High-Grade Gliomas). In: QuiñonesHinojosa, A. \& Raza, S. (Eds.). Controversies in Neuro-Oncology. New York, Thieme, 2014.

Kraus, J. A.; Glesmann, N.; Beck, M.; Krex, D.; Klockgether, T.; Schackert, G. \& Schlegel, U. Molecular analysis of the PTEN, TP53 and CDKN2A tumor suppressor genes in long-term survivors of glioblastoma multiforme. J. Neurooncol., 48(2):89-94, 2000.

Lee, C. H,; Jung, K. W.; Yoo, H.; Park, S. \& Lee, S. H. Epidemiology of primary brain and central nervous system tumors in Korea. J. Korean Neurosurg. Soc., 48(2):145-52, 2010.

Louis, D. N.; Ohgaki, H.; Wiestler, O. D.; Cavenee, W. K.; Burger, P. C.; Jouvet, A.; Scheithauer, B. W. \& Kleihues, P. The 2007 WHO classification of tumours of the central nervous system. Acta Neuropathol., 114(2):97-109, 2007.

Ohgaki, H. \& Kleihues, P. Population-based studies on incidence, survival rates, and genetic alterations in astrocytic and oligodendroglial gliomas. J. Neuropathol. Exp. Neurol., 64(6):479-89, 2005.

Ohgaki, H.; Dessen, P.; Jourde, B.; Horstmann, S.; Nishikawa, T.; Di Patre, P. L.; Burkhard, C.; Schüler, D.; Probst-Hensch, N. M.; Maiorka, P. C.; et al. Genetic pathways to glioblastoma: a population-based study. Cancer Res., 64(19):6892-9, 2004.

Ostrom, Q. T.; Bauchet, L.; Davis, F. G.; Deltour, I; Fisher, J. L.; Langer, C. E.; Pekmezci, M.; Schwartzbaum, J. A.; Turner, M. C.; Walsh, K. M.; et al. The epidemiology of glioma in adults: a "state of the science" review. Neuro Oncol., 16(7):896-913, 2014.

Ostrom, Q. T.; Gittleman, H.; Farah, P.; Ondracek, A.; Chen, Y.; Wolinsky, Y.; Stroup, N. E.; Kruchko, C. \& Barnholtz-Sloan, J. S. CBTRUS statistical report: Primary brain and central nervous system tumors diagnosed in the United States in 2006-2010. Neuro Oncol., 15 Suppl. 2:ii1-56, 2013.

Smith, J. S.; Tachibana, I.; Passe, S. M.; Huntley, B. K.; Borell, T. J.; Iturria, N.; O'Fallon, J. R.; Schaefer, P. L.; Scheithauer, B. W.; James, C. D.; et al. PTEN mutation, EGFR amplification, and outcome in patients with anaplastic astrocytoma and glioblastoma multiforme. J. Natl. Cancer Inst., 93(16):1246-56, 2001.

Srividya, M. R.; Thota, B.; Shailaja, B. C.; Arivazhagan, A.; Thennarasu, K.; Chandramouli, B. A.; Hegde, A. S. \& Santosh, V. Homozygous 10q23/PTEN deletion and its impact on outcome in glioblastoma: a prospective translational study on a uniformly treated cohort of adult patients. Neuropathology, 31(4):376-83, 2011.

Stupp, R.; Mason, W. P.; van den Bent, M. J.; Weller, M.; Fisher, B.; Taphoorn, M. J.; Belanger, K.; Brandes, A. A.; Marosi, C.; Bogdahn, U.; et al. Radiotherapy plus concomitant and adjuvant temozolomide for glioblastoma. N. Engl. J. Med., 352(10):987-96, 2005.

Umesh, S.; Tandon, A.; Santosh, V.; Anandh, B.; Sampath, S.; Chandramouli, B. A. \& Sastry Kolluri, V. R. Clinical and immunohistochemical prognostic factors in adult glioblastoma patients. Clin. Neuropathol., 28(9):362-72, 2009.

Xu, J.; Li, Z.; Wang, J.; Chen, H. \& Fang, J. Y. Combined PTEN mutation and protein expression associate with overall and disease-free survival of glioblastoma patients. Transl. Oncol., 7(2):196-205, 2014.

Yamada, K. M. \& Araki, M. Tumor suppressor PTEN: modulator of cell signaling, growth, migration and apoptosis. J. Cell Sci., 114(Pt. 13):2375-82, 2001

Yang, L.; Clarke, M. J.; Carlson, B. L.; Mladek, A. C.; Schroeder, M. A.; Decker, P.; Wu, W.; Kitange, G. J.; Grogan, P. T.; Goble, J. M.; et al. PTEN loss does not predict for response to RAD001 (Everolimus) in a glioblastoma orthotopic xenograft test panel. Clin. Cancer Res., 14(12):3993-4001, 2008.

\author{
Dirección para correspondencia \\ Esteban Fabián Espinoza García \\ Salinas 1392, casilla 150 \\ San Felipe \\ CHILE
}

Email: esteban.espinoza@postgrado.uv.cl

Recibido : 11-10-2018

Aceptado :12-03-2019 